\title{
Sorafenib in Relapsed AML With FMS-Like Receptor Tyrosine Kinase-3 Internal Tandem Duplication Mutation
}

\author{
Smith Giri, MBBS ${ }^{a}$; Shadi Hamdeh, MD'; Vijaya Raj Bhatt, MBBSc; and James K. Schwarz, MDc
}

\begin{abstract}
Old age ( $\leq 65$ years), relapsed or refractory disease, and the presence of FMS-like receptor tyrosine kinase-3 (FLT3) internal tandem duplication (ITD) mutation are poor prognostic factors in acute myeloid leukemia (AML). FLT3 inhibitors such as sorafenib have been shown to have a potential role in treating relapsed or refractory AML with FLT3 mutations. In the present report, the use of sorafenib in combination with cytarabine and idarubicin resulted in disease control for 7 months in an older patient with relapsed FLT3-positive AML. This case report and the existing literature indicate that sorafenib has disease activity against relapsed AML with the FLT3-ITD mutation in older patients. Larger multicenter studies should be conducted to confirm these findings, which have the potential to improve outcomes in this high-risk AML subgroup. (J Natl Compr Canc Netw 2015;13:508-514)
\end{abstract}

\section{NCCN: Continuing Education}

\section{Accreditation Statement}

This activity has been designated to meet the educational needs of physicians and nurses involved in the management of patients with cancer. There is no fee for this article. No commercial support was received for this article. The National Comprehensive Cancer Network (NCCN) is accredited by the ACCME to provide continuing medical education for physicians.

\footnotetext{
From the ad Department of Medicine, University of Tennessee Health Science Center, Memphis, Tennessee; and bepartment of Internal Medicine, Creighton University Medical Center, and 'Department of Internal Medicine, Division of Hematology and Oncology, University of Nebraska Medical Center, Omaha, Nebraska.

Submitted July 7, 2014; accepted for publication October 2, 2014.

The authors have disclosed that they have no financial interests, arrangements, affiliations, or commercial interests with the manufacturers of any products discussed in this article or their competitors.

Correspondence: Vijaya Raj Bhatt, MBBS, University of Nebraska Medical Center, Department of Internal Medicine, Division of HematologyOncology, 987680 Nebraska Medical Center, Omaha, NE 68198-7680.

E-mail: vijaya.bhatt@unmc.edu
}

NCCN designates this journal-based CME activity for a maximum of 1.0 AMA PRA Category 1 Credit(s) TM. Physicians should claim only the credit commensurate with the extent of their participation in the activity.

NCCN is accredited as a provider of continuing nursing education by the American Nurses Credentialing Center's Commission on Accreditation.

This activity is accredited for 1.0 contact hours. Accreditation as a provider refers to recognition of educational activities only; accredited status does not imply endorsement by NCCN or ANCC of any commercial products discussed/displayed in conjunction with the educational activity. Kristina M. Gregory, RN, MSN, OCN, is our nurse planner for this educational activity.

All clinicians completing this activity will be issued a certificate of participation. To participate in this journal CE activity: 1) review the learning objectives and author disclosures; 2 ) study the education content; 3) take the posttest with a $66 \%$ minimum passing score and complete the evaluation at http://education.nccn.org/ node/65994; and 4) view/print certificate.

Release date: May 13, 2015; Expiration date: May 13, 2016

\section{Learning Objectives}

Upon completion of this activity, participants will be able to:

- Describe the factors associated with poor prognosis for patients with AML

- Discuss the potential benefits of the use of several therapies to treat patients with FLT3-ITD mutations

\section{EDITOR}

Kerrin M. Green, MA, Assistant Managing Editor, JNCCN_Journal of the National Comprehensive Cancer Network

Ms. Green has disclosed that she has no relevant financial relationships.

\section{CE AUTHORS}

Deborah J. Moonan, RN, BSN, Director, Continuing Education, has disclosed that she has no relevant financial relationships.

Ann Gianola, MA, Manager, Continuing Education Accreditation \& Program Operations, has disclosed that she has no relevant financial relationships.

Kristina M. Gregory, RN, MSN, OCN, Vice President, Clinical Information Operations, has disclosed that she has no relevant financial relationships. Rashmi Kumar, PhD, Senior Manager, Clinical Content, has disclosed that she has no relevant financial relationships.

Courtney Smith, PhD, Oncology Scientist/Medical Writer, has disclosed that she has no relevant financial relationships. 
Acute myeloid leukemia (AML) is the most common form of acute leukemia in adults, with an estimated incidence of 3 cases per 100,000 people. ${ }^{1}$ Factors associated with poor prognosis include advanced age, unfavorable cytogenetics, molecular markers (such as FMS-like receptor tyrosine kinase-3 [FLT3], internal tandem duplication [ITD] mutation, or absence of nucleophosmin [NPM1] mutation), poor performance status, multiple comorbidities, inability to tolerate chemotherapy, and multidrug resistance. ${ }^{2}$ The management of patients with relapsed/refractory AML remains a huge clinical challenge with few therapeutic options available, particularly for older patients. ${ }^{3}$

Among the intermediate cytogenetic risk group with normal karyotype, the FLT3-ITD mutation portends poor disease-free and overall survivals. ${ }^{4}$ FLT3 is normally expressed on the surface of hematopoietic stem cells and is important in the normal development of pluripotent stem cells. ${ }^{5}$ FLT3 is mutated in approximately one-third of AML cases. ${ }^{4}$ Therapeutic options in cases of refractory or relapsed FLT3-positive AML are limited, particularly in the presence of poor performance status or prior allogenic stem cell transplantation (alloSCT). ${ }^{3}$ FLT3 inhibitors, such as sorafenib, may have some role in these situations. ${ }^{6}$ Sorafenib was initially designed as a small molecule inhibitor of c-Raf kinase but has demonstrated efficacy in inhibiting the activities of FLT3, vascular endothelial growth factor receptors 2 and 3 , and members of the platelet-derived growth factor receptor family. ${ }^{7}$ This report presents a case of an older man with relapsed FLT3-positive AML who experienced disease control for 7 months with the combination of chemotherapy and sorafenib.

\section{Case Presentation}

A 65-year-old man presented to the emergency room who had experienced worsening pain in both calves during the past 2 to 3 weeks, fever and chills for 1 week, rectal bleeding, and weight loss. His past medical history was significant for prostate adenocarcinoma (Gleason 7) treated 4 years ago with intensity-modulated radiation therapy (8000 cGy in 40 fractions), diabetes mellitus, hypertension, hyperlipidemia, atrial fibrillation, gastroesophageal reflux disease complicated by Barrett esophagus, and glaucoma. Family history was significant for head and neck cancer in his father. The patient denied smoking or drinking alcohol. Physical examination revealed a blood pressure reading of $130 / 70 \mathrm{~mm} \mathrm{Hg}$, heart rate of 77 beats per minute, temperature of $98.6^{\circ} \mathrm{F}$, respiratory rate of 18 breaths per minute, and oxygen saturation of $96 \%$. He had bilateral calf tenderness without erythema or edema. The remainder of the examination was unremarkable.

Laboratory evaluation revealed a WBC count of $59,000 / \mathrm{mcL}$ with $93 \%$ blasts, hemoglobin level of $9.2 \mathrm{~g} / \mathrm{dL}$, platelet count of $83,000 / \mathrm{mcL}$ (Table 1), and normal glucose and electrolyte levels, coagulation profile, and renal and liver function tests. Bone marrow biopsy revealed blasts with high nuclear-to-cytoplasmic ratio, occasional nucleoli, and some folded or bilobed nuclei. Most blasts appeared agranular; however, rare cytoplasmic granules and Auer rods were seen. Blasts expressed CD33, CD45, partial CD38, partial CD71, partial CD117, and cytoplasmic myeloperoxidase, consistent with AML. The blasts were negative for CD34, HLA-DR, terminal deoxynucleotidyl transferase, and lymphoid markers. Cytogenetics demonstrated normal male chromosomes, 46,XY[20]. Fluorescence in situ hybridization was negative for $\mathrm{t}(15 ; 17)$ translocation. Deoxyribonucleic acid amplification showed FLT-ITD and NPM1 mutations.

The patient received induction chemotherapy with 7 -day infusional cytarabine $\left(100 \mathrm{mg} / \mathrm{m}^{2} / \mathrm{d}\right)$ and 3 -day daunorubicin $\left(60 \mathrm{mg} / \mathrm{m}^{2} / \mathrm{d}\right)$. A repeat bone marrow biopsy at day 14 demonstrated residual leukemia with $15 \%$ blasts. After a lengthy discussion regarding different options, including alloSCT in the future, the patient chose to be treated with low-intensity therapy. Hence, he was started on subcutaneous azacytidine $\left(75 \mathrm{mg} / \mathrm{m}^{2} / \mathrm{d}\right.$ for 7 days) with a repeat cycle every 4 weeks. The patient received 9 cycles of azacytidine, and showed an improvement in his hemogram.

Ten months after his first presentation, the patient presented with dyspepsia and was feeling unwell. His CBC count was $20,700 / \mathrm{mcL}$ with $80 \%$ blasts. The patient was started on intermediatedose cytarabine $\left(1.5 \mathrm{~g} / \mathrm{m}^{2} / \mathrm{d}\right.$ for 3 days $)$, idarubicin (12 $\mathrm{mg} / \mathrm{m}^{2} / \mathrm{d}$ for 3 days), and sorafenib (400 mg twice daily for 7 days). ${ }^{8}$ The patient also received prophylactic filgrastim and prophylactic antibiotic, antifungal, and antiviral agents. A repeat bone marrow biopsy at day 28 showed 50\% cellularity and $5 \%$ blasts. Cytogenetics demonstrated normal male chromosomes, 46,XY[19]. The patient tolerated 
Giri et al

Table 1 Hemogram Results During Different Stages of Disease

\begin{tabular}{|c|c|c|c|c|}
\hline & WBC (per mcL) & Blasts (\% of WBC) & $\begin{array}{l}\text { Hemoglobin } \\
(\mathrm{g} / \mathrm{dL})\end{array}$ & $\begin{array}{l}\text { Platelet Count } \\
\text { (per mcL) }\end{array}$ \\
\hline At diagnosis & 59,000 & $93 \%$ & 9.2 & 83,000 \\
\hline $\begin{array}{l}\text { After } 1 \text { month of first induction } \\
\text { therapy with cytarabine and } \\
\text { daunorubicin }\end{array}$ & 1700 & $0 \%$ & 10.1 & 185,000 \\
\hline Before 9th cycle of azacytidine & 3300 & $0 \%$ & 14.4 & 307,000 \\
\hline At first relapse & 20,700 & $80 \%$ & 14.7 & 158,000 \\
\hline $\begin{array}{l}\text { After } 1 \text { month of second } \\
\text { induction with idarubicin, } \\
\text { cytarabine, and sorafenib }\end{array}$ & 5200 & $3 \%$ & 10.1 & 416,000 \\
\hline $\begin{array}{l}\text { After } 1 \text { month of consolidation } \\
\text { with idarubicin, cytarabine, and } \\
\text { sorafenib }\end{array}$ & 5400 & $0 \%$ & 11.8 & 245,000 \\
\hline One month before second relapse & 5800 & $0 \%$ & 15.0 & 194,000 \\
\hline At second relapse & 110,000 & $93 \%$ & 11.9 & 16,000 \\
\hline
\end{tabular}

induction chemotherapy well, experiencing grade 1 skin rash, grade 1 transaminitis, and one episode of neutropenic fever. However, he presented 2 weeks after discharge with pleuritic chest pain and was found to have pulmonary embolism. This was managed with outpatient therapy with enoxaparin. The patient subsequently received one cycle of consolidation therapy with cytarabine, $0.75 \mathrm{~g} / \mathrm{m}^{2} / \mathrm{d}$ for 3 days; idarubicin, $8 \mathrm{mg} / \mathrm{m}^{2} / \mathrm{d}$ for 2 days; and sorafenib, $400 \mathrm{mg}$ twice daily for 28 days. ${ }^{8}$ This was complicated by prolonged myelosuppression, sepsis, clostridium difficile infection, weakness, and anorexia. On recovery, the patient was maintained on single-agent sorafenib, $400 \mathrm{mg}$ twice daily, ${ }^{8}$ which was well tolerated. Seven months after the first relapse, he experienced disease relapse with elevation of his WBC count to $110,000 / \mathrm{mcL}$ with $93 \%$ blasts. At this point, he was started on hydroxyurea, lowdose cytarabine, and sorafenib, $400 \mathrm{mg}$ twice daily. The patient expired 3 months after his second relapse and 17 months after his initial diagnosis.

\section{Discussion}

The presence of an FLT3-ITD mutation in AML has been associated with higher rates of relapse and shorter disease-free and overall survivals. ${ }^{9}$ A previous study had reported a relapse rate of $74 \%$ and a 5 -year overall survival rate of $32 \%$ in individuals with these mutations. ${ }^{10}$ The outcomes are significantly worse in patients with FLT3-mutated AML with relapsed disease, advanced age, or poor performance status. However, in patients with NPM1-mutated AML, the presence of low-allelic burden FLT3ITD mutation, compared with wild-type FLT3, does not confer poor outcomes. ${ }^{11}$ Although this patient had an NPM1 mutation, the allelic burden of the FLT3-ITD mutation was not available. Increasing recognition of the FLT3-ITD mutation as an adverse prognostic factor in patients with AML has led to the development of potent tyrosine kinase inhibitors targeting this mutation. Several FLT3 inhibitors, such as sorafenib, quizartinib, crenolanib, midostaurin, lestaurtinib, sunitinib, and tandutinib, have shown promising results in these subgroups of patients. ${ }^{5}$ Limited phase I/II studies on the different FLT3 inhibitors have suggested a potential benefit of these agents; however, benefits had a short duration in most of these studies. ${ }^{8,12-16}$ The long-term utility of these agents has been hampered by the development of drug resistance. Possible mechanisms of drug resistance include point mutations in the FLT3 tyrosine kinase domain, upregulation of the antiapoptotic protein MCL-1, or constitutive activation of FLT3. ${ }^{17}$ Newer-generation FLT3ITD inhibitors may be effective in these patients. Newer agents such as crenolanib and quizartinib, individually or in combination with sorafenib, have been shown to be useful in such resistant cases. ${ }^{18-20}$ Similarly, ponatinib is a multikinase inhibitor that has been shown to be effective in cases of acquired FLT3 tyrosine kinase domain mutations. ${ }^{21,22}$ 
Sorafenib has been tested in patients with AML both as a monotherapy and in combination with other chemotherapies (Table 2). Pratz et al ${ }^{23}$ conducted a phase I dose-escalation trial evaluating sorafenib as a monotherapy in relapsed/refractory advanced leukemia. Although marked FLT3-ITD inhibition was achieved, the clinical response was only modest, with 11 of 15 patients experiencing stable disease as the best response. Metzelder et $\mathrm{al}^{6}$ studied the compassionate use of sorafenib among 6 cases of patients with relapsed/refractory FLT3-ITD mutated AML before and after alloSCT. Sorafenib induced clinically meaningful responses (ranging from a hematologic response to complete remission) in $100 \%$ of patients. Similarly, Zhang et $\mathrm{al}^{7}$ reported a clinical response in all 6 patients, who carried solely an FLT3-ITD mutation. A marked reduction in median percentage of blasts in peripheral blood and bone marrow was reported after initiating sorafenib monotherapy in these patients (reduction from $81 \%$ to $7 \%$ in peripheral blood, and from $75 \%$ to $34 \%$ in bone marrow, respectively). ${ }^{7}$ Similarly, a phase I doseescalation study of sorafenib monotherapy in patients with refractory/relapsed acute leukemia showed complete remission in 5 of 50 patients (10\%), but in all patients (100\%) with FLT3-ITD mutations. ${ }^{24}$ Hence, sorafenib has single-agent activity in patients with relapsed/refractory AML with FLT3-ITD mutations.

Few studies have investigated the role of sorafenib in combination with chemotherapy in patients with AML. Inaba et $\mathrm{a}^{25}$ conducted a phase I pharmacokinetic and pharmacodynamic study of sorafenib in combination with clofarabine and cytarabine in pediatric relapsed/refractory leukemia. Overall response was seen in 9 of 11 patients $(82 \%)$, but in all 5 patients with FLT3-ITD mutations (100\%). A dose of $150 \mathrm{mg} / \mathrm{m}^{2}$ seemed to be safer than a dose of 200 $\mathrm{mg} / \mathrm{m}^{2}$, with no dose-limiting toxicities noted with the former dosing. In a phase I/II study of sorafenib in combination with cytarabine and idarubicin (regimen used in this case report), a complete remission rate of $75 \%$ and $93 \%$ was achieved among the entire cohort and patients with FLT3-mutated AML. This resulted in the probability of survival of $74 \%$ at 1 year, thus suggesting a high response rate with the combination of sorafenib with chemotherapy in patients with FLT3-mutated AML. ${ }^{8}$ Another phase I/ II study of sorafenib in combination with low-dose cytarabine in older patients, otherwise unsuitable for intensive chemotherapy, showed an overall response of $10 \%$. This could be explained by underrepresentation of FLT3-ITD mutations in the study population, which was only seen in 3 of 21 patients $(14 \%){ }^{26}$ Another phase II trial tested the combination of azacytidine and sorafenib in 37 patients with refractory/relapsed AML with FLT3-ITD mutations. The overall response rate was $46 \%$, with $64 \%$ achieving greater than $85 \%$ FLT3 inhibition during the first cycle of chemotherapy. ${ }^{27} \mathrm{~A}$ recent randomized controlled trial showed that the addition of sorafenib to cytarabine and daunorubicin is not beneficial among older patients with AML (including in a subgroup with FLT3-ITD mutations). Because the trial included only 28 patients with FLT3-ITD mutations, the study may not have adequate power to detect the difference in outcomes in this subgroup. ${ }^{28}$

The optimal role of sorafenib in patients with AML remains unanswered. For example, sorafenib monotherapy has not been compared with the combination of sorafenib and chemotherapy in patients with AML and the FLT3-ITD mutation, and therefore the role of combination therapy versus sorafenib monotherapy remains unclear. Despite promising results of sorafenib in these clinical trials, the development of resistance during the course of therapy is a major clinical challenge. Given their short-term efficacy and relatively few adverse effects, these agents seem to be particularly helpful as monotherapy in medically unfit patients with relapsed/refractory AML with FLT3-ITD mutations, who otherwise are poor candidates to receive cytotoxic chemotherapy (because of prior therapy). Thus, in select patients, sorafenib may be used as a bridge to alloSCT. ${ }^{29}$ The 2015 NCCN Clinical Practice Guidelines in Oncology (NCCN Guidelines) for AML acknowledge the poor prognosis associated with the presence of FLT3-ITD mutations in patients with AML with normal karyotype. Although data are considered inadequate to support the use of sorafenib during induction, it may be considered in relapsed AML. Importantly, NCCN Guidelines recommend clinical trials as the standard therapy for patients with FLT3 abnormalities or other poor prognostic features. (to view the most recent version of these guidelines, visit NCCN.org). ${ }^{30}$ Conversely, in clinical practice, sorafenib is frequently used as an off-label indication in patients with relapsed/refractory AML with the FLT3-ITD mutation. ${ }^{29}$ 


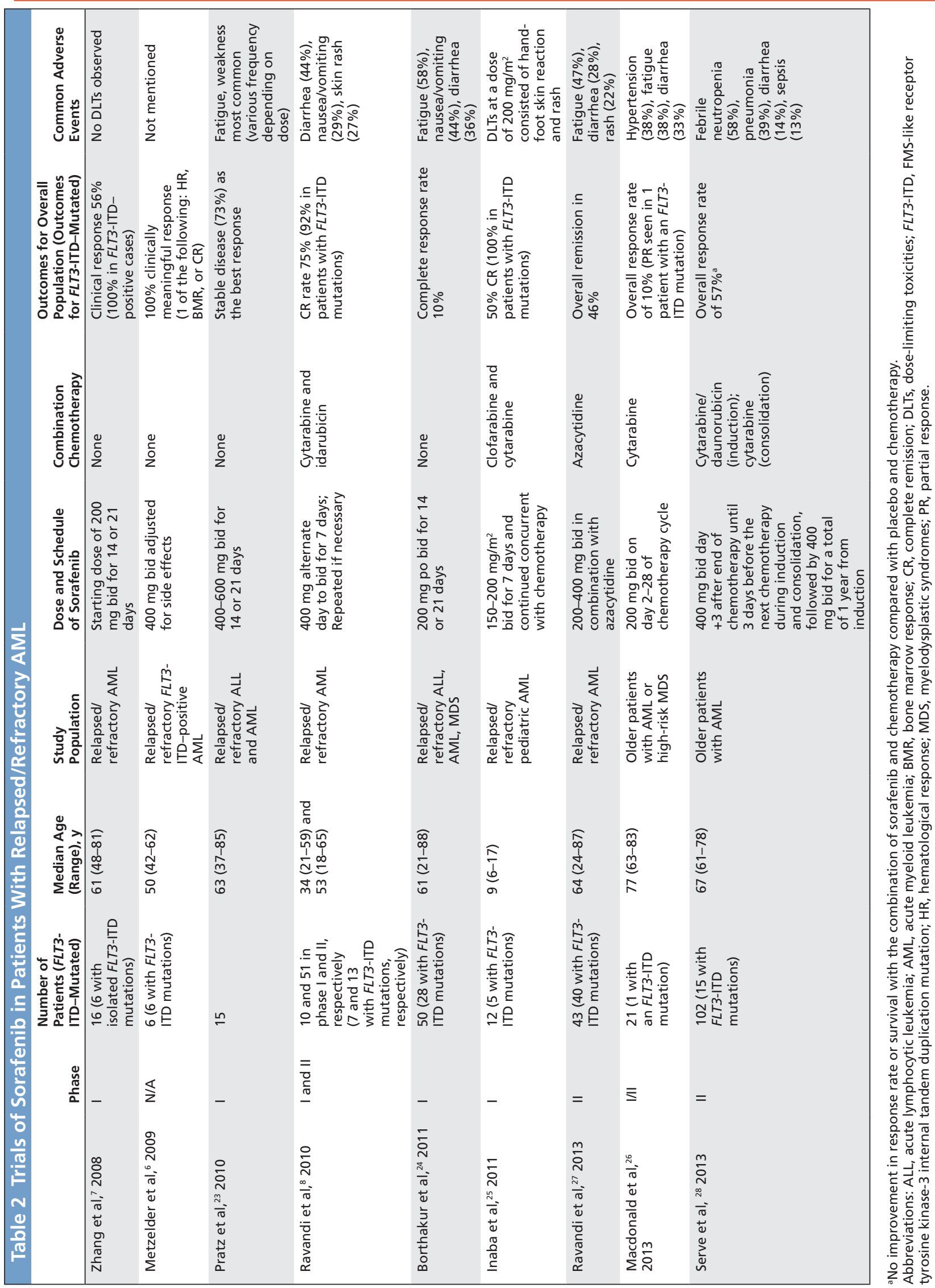


The present patient received initial standard chemotherapy with cytarabine and daunorubicin, followed by azacytidine. Although this patient was a potential candidate for alloSCT, he declined this option. Relapse at 10 months was managed with sorafenib in combination with cytarabine and idarubicin, followed by consolidation therapy and sorafenib maintenance, which resulted in disease control for 7 months. The response duration of 7 months in an older patient with relapsed AML and the FLT3-ITD mutation indicates a good response to the sorafenib-based therapy. This patient was able to tolerate induction therapy without major toxicity but developed complications during consolidation therapy. Hence, he was maintained on single-agent sorafenib. Several studies discussed previously ${ }^{6-8,25}$ demonstrated a role for sorafenib in patients with relapsed/refractory AML and the FLT3-ITD mutation. Sorafenib monotherapy is often easily tolerated, even by heavily pretreated older patients, ${ }^{6,7,23,24}$ as illustrated in this case. Hence, it can be used as a palliative option or a bridge to alloSCT in eligible patients. ${ }^{29}$ Larger phase II/III trials are indicated to compare the outcomes of sorafenib monotherapy versus its combination with chemotherapy in patients with relapsed/refractory AML with the FLT3-ITD mutation. Future studies should also focus on management of AML in patients who develop resistance to first-line FLT3 inhibitors.

\section{References}

1. Sant M, Allemani C, Tereanu C, et al. Incidence of hematologic malignancies in Europe by morphologic subtype: results of the HAEMACARE project. Blood 2010;116:3724-3734.

2. Estey EH. Acute myeloid leukemia: 2013 update on risk-stratification and management. Am J Hematol 2013;88:318-327.

3. Metzelder SK, Wollmer E, Neubauer A,rchert A. Sorafenib in relapsed and refractory FLT3-ITD positive acute myeloid leukemia: a novel treatment option [in German]. Dtsch Med Wochenschr 2010;135:1852-1856.

4. Mattison RJ, Ostler KR, Locke FL, Godley LA. Implications of FLT3 mutations in the therapy of acute myeloid leukemia. Rev Recent Clin Trials 2007;2:135-141.

5. Wiernik PH. FLT3 inhibitors for the treatment of acute myeloid leukemia. Clin Adv Hematol Oncol 2010;8:429-436, 444

6. Metzelder S, Wang Y, Wollmer E, et al. Compassionate use of sorafenib in FLT3-ITD-positive acute myeloid leukemia: sustained regression before and after allogeneic stem cell transplantation. Blood 2009;113:6567-6571.

7. Zhang W, Konopleva M, Shi YX, et al. Mutant FLT3: a direct target of sorafenib in acute myelogenous leukemia. J Natl Cancer Inst 2008;100:184-198.

8. Ravandi F, Cortes JE, Jones D, et al. Phase I/II study of combination therapy with sorafenib, idarubicin, and cytarabine in younger patients with acute myeloid leukemia. J Clin Oncol 2010;28:1856-1862.

9. Swords R, Freeman C, Giles F. Targeting the FMS-like tyrosine kinase 3 in acute myeloid leukemia. Leukemia 2012;26:2176-2185.

10. Kottaridis PD, Gale RE, Frew ME, et al. The presence of a FLT3 internal tandem duplication in patients with acute myeloid leukemia (AML) adds important prognostic information to cytogenetic risk group and response to the first cycle of chemotherapy: analysis of 854 patients from the United Kingdom Medical Research Council AML 10 and 12 trials. Blood 2001;98:1752-1759.

11. Pratcorona M, Brunet $S$, Nomdedeu J, et al. Favorable outcome of patients with acute myeloid leukemia harboring a low-allelic burden FLT3-ITD mutation and concomitant NPM1 mutation: relevance to post-remission therapy. Blood 2013;121:2734-2738.

12. Fiedler W, Serve H, Dohner H, et al. A phase 1 study of SU11248 in the treatment of patients with refractory or resistant acute myeloid leukemia (AML) or not amenable to conventional therapy for the disease. Blood 2005;105:986-993.

13. Fischer T, Stone RM, Deangelo DJ, et al. Phase IIB trial of oral midostaurin (PKC412), the FMS-like tyrosine kinase 3 receptor (FLT3) and multitargeted kinase inhibitor, in patients with acute myeloid leukemia and high-risk myelodysplastic syndrome with either wild-type or mutated FLT3. J Clin Oncol 2010;28:4339-4345.

14. Knapper S, Burnett AK, Littlewood T, et al. A phase 2 trial of the FLT3 inhibitor lestaurtinib (CEP701) as first-line treatment for older patients with acute myeloid leukemia not considered fit for intensive chemotherapy. Blood 2006;108:3262-3270.

15. Smith BD, Levis M, Beran M, et al. Single-agent CEP-701, a novel FLT3 inhibitor, shows biologic and clinical activity in patients with relapsed or refractory acute myeloid leukemia. Blood 2004;103:3669-3676.

16. Stone RM, DeAngelo DJ, Klimek V, et al. Patients with acute myeloid leukemia and an activating mutation in FLT3 respond to a small-molecule FLT3 tyrosine kinase inhibitor, PKC412. Blood 2005;105:54-60.

17. Weisberg E, Barrett R, Liu Q, et al. FLT3 inhibition and mechanisms of drug resistance in mutant FLT3-positive AML. Drug Resist Updat 2009; 12:81-89.

18. Galanis A, Ma H, Rajkhowa $T$, et al. Crenolanib is a potent inhibitor of FLT3 with activity against resistance-conferring point mutants. Blood 2014;123:94-100.

19. Kampa-Schittenhelm KM, Heinrich MC, Akmut F, et al. Quizartinib (AC220) is a potent second generation class III tyrosine kinase inhibitor that displays a distinct inhibition profile against mutant-FLT3, -PDGFRA and -KIT isoforms. Mol Cancer 2013;12:19.

20. Zhang W, Gao C, Konopleva M, et al. Reversal of acquired drug resistance in FLT3-mutated acute myeloid leukemia cells via distinct drug combination strategies. Clin Cancer Res 2014 2014;20:2363-2374.

21. Zirm E, Spies-Weisshart B, Heidel F, et al. Ponatinib may overcome resistance of FLT3-ITD harbouring additional point mutations, notably the previously refractory F691I mutation. Br J Haematol 2012;157:483-492.

22. Smith CC, Lasater EA, Zhu X, et al. Activity of ponatinib against clinically-relevant AC220-resistant kinase domain mutants of FLT3-ITD. Blood 2013;121:3165-3171.

23. Pratz KW, Cho E, Levis MJ, et al. A pharmacodynamic study of sorafenib in patients with relapsed and refractory acute leukemias. Leukemia 2010;24:1437-1444.

24. Borthakur G, Kantarjian H, Ravandi F, et al. Phase I study of sorafenib in patients with refractory or relapsed acute leukemias. Haematologica 2011;96:62-68.

25. Inaba H, Rubnitz JE, Coustan-Smith E, et al. Phase I pharmacokinetic and pharmacodynamic study of the multikinase inhibitor sorafenib in combination with clofarabine and cytarabine in pediatric relapsed/ refractory leukemia. J Clin Oncol 2011;29:3293-3300.

26. Macdonald DA, Assouline SE, Brandwein J, et al. A phase I/II study of sorafenib in combination with low dose cytarabine in elderly patients with acute myeloid leukemia or high-risk myelodysplastic syndrome from the National Cancer Institute of Canada Clinical Trials Group: trial IND.186. Leuk lymphoma 2013;54:760-766.

27. Ravandi F, Alattar ML, Grunwald MR, et al. Phase 2 study of azacytidine plus sorafenib in patients with acute myeloid leukemia and FLT-3 internal tandem duplication mutation. Blood 2013;121:4655-4662.

28. Serve H, Krug U, Wagner R, et al. Sorafenib in combination with intensive chemotherapy in elderly patients with acute myeloid leukemia: results from a randomized, placebo-controlled trial. J Clin Oncol 2013;31:3110-3118.

29. Sammons SL, Pratz KW, Smith BD, et al. Sorafenib is tolerable and improves clinical outcomes in patients with FLT3-ITD acute myeloid leukemia prior to stem cell transplant and after relapse post-transplant. Am J Hematol 2014;89:936-938.

30. O'Donnell MR, Tallman MS, Abboud CN, et al. NCCN Clinical Practice Guidelines in Oncology: Acute Myeloid Leukemia. Version 1.2015. Available at: NCCN.org. Accessed April 17, 2015. 
Giri et al

\section{Instructions for Completion}

To participate in this journal CE activity: 1) review the learning objectives and author disclosures; 2 ) study the education content; 3 ) take the posttest with a $66 \%$ minimum passing score and complete the evaluation at http://education.nccn.org/ node/65994; and 4) view/print certificate. After reading the article, you should be able to answer the following multiple- choice questions. Credit cannot be obtained for tests completed on paper. You must be a registered user on NCCN.org. If you are not registered on NCCN.org, click on "New Member? Sign up here" link on the left hand side of the Web site to register. Only one answer is correct for each question. Once you successfully answer all posttest questions you will be able to view and/or print your certificate. Software requirements: Internet.

\section{Posttest Questions}

1. FLT3-ITD mutation is a frequent molecular abnormality noted in approximately one-third of adults with AML. Which of the following statements is NOT true about FLT3 or AML with the FLT3-ITD mutation?

a. FLT3 is normally expressed on the surface of hematopoietic stem cells and is important in the normal development of pluripotent stem cells.

b. Among adult patients with AML with normal karyotype, the presence of the FLT3-ITD mutation, compared with wild-type $F L T 3$, portends a worse overall survival.

c. FLT-3 inhibitors such as sorafenib have activity against AML with the FLT3-ITD mutation; however, the longterm utility of these agents has been hampered by the development of drug resistance.

d. In patients with AML with NPM1 mutation, the presence of a high-allelic burden FLT3-ITD mutation, compared with wild-type FLT3, does not confer worse outcomes.
2. FLT3 inhibitor studies using sorafenib suggest:

a. Single-agent sorafenib is preferred to combination chemotherapy followed by allogeneic hematopoietic stem cell transplant in the management of patients with relapsed AML with the FLT3-ITD mutation

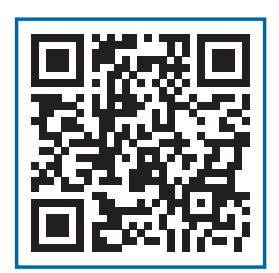

b. Promise as a therapeutic option because of the long duration of response in most studies

c. Drug resistance may result from point mutations in the FLT3 tyrosine kinase domain, upregulation of MCL-1, or constitutive FLT3 activation

d. Single-agent sorafenib is not recommended for medically unfit patients with AML with the FLT3-ITD mutation

3. True or False: Sorafenib in combination with other chemotherapy has been studied in both adult and pediatric patients with the FLT3-ITD mutation. 